\title{
SOLUTION-FOCUSED BRIEF THERAPY AND SUICIDE PREVENTION ${ }^{1)}$
}

\author{
Heather Fiske, Ph.D.
}

Solution-Focused Brief Therapy (SFBT) is an efficient and effective approach to clinical work with individuals who are at risk of suicide, and their families (Fiske, 2008). One reason that SFBT is time-effective is that it utilizes strengths and capacities already present in clients, their families and friends, and their communities. Also, highlighting those strengths and capacities is hope-inducing for the people involved.

There are other good reasons for using SFBT, among them the ways in which solution-focused conversation helps to focus both client and therapist on hope and reasons for living. This focus reduces the amount of time spent going over clients' thoughts and plans for suicide. According to Thomas Joiner's psychological-interactional theory of suicide, people acquire the ability to kill themselves partly by "rehearsing" and thereby desensitizing suicidal action (Joiner, 2005). SFBT selectively amplifies strengths, resources, and solutions, making such desensitization less likely.

Solution-focused practice is client-focused, which mitigates the chance of clients feeling that they are being subjected to a formula. Such impressions are especially likely with the use of standardized risk assessment and no-harm-contracting protocols.

Scaling and other solution-focused practices help clients to break both problems and change into smaller, more manageable steps ("partializing"). This process undermines beliefs that one's problems are interminable, inescapable, and intolerable-beliefs that push clients toward suicide as a solution.

Individuals who are focused on suicide as a solution often have difficulty imagining any personal future. A unique contribution of SFBT is its emphasis on developing a detailed picture of a preferred future, using tools such as the miracle question. This is one way in which solution-focused conversation is a "tap on the shoulder", drawing clients' attention to possibilities they could not access on their own.

For therapists, attending to clients' strengths and focusing on hope and reasons for living can act as both prevention and antidote for secondary trauma.

\section{Application: Solution-Focused Questions}

(Note: All of the questions mentioned here are "second-rate", because they are generic; the best questions are based on what a particular client says.) 
What are your best hopes for this conversation? For desperate clients, this opening question often strikes to the heart of the matter: the struggle for hope and reasons for living. It makes sense to them, and is a way of beginning to determine the destination of treatment.

Suppose a miracle happened, and (those best hopes)...were realized, what is the first small sign you would notice...? Again, this question often makes sense because it fits with the clients' beliefs (that it would take a miracle to change things).

What would your best friend (or other close person) say? Relationship questions provide alternative viewpoints and information on supportive connections.

When was the most recent exception to the problem-or (better)

When was the most recent instance of a solution/part of the miracle picture?

Instances of solution challenge the hegemony of problems.

On a scale from 1 to $10 \ldots$ Scaling questions can yield quick information about client safety, goals, and progress. Also, they "partialize", and may engage more cognitive resources in emotion-laden decision-making.

What else? Details of strengths and resources, exceptions or instances, make them real and live for clients. Details of a miracle picture create "future pull", motivating clients to move toward those futures.

\section{$\underline{\text { Stance }}$}

More important than any question or technique is the solution-focused stance: respectful, curious, mindful.

\section{REFERENCES}

Fiske, H. (2008). Hope in action: Solution-focused conversations about suicide. New York: Routledge.

Joiner, T. (2005). Why people die by suicide. Cambridge, MA: Harvard University Press.

1) Presented at the Eighth Conference of the National Foundation of Brief Therapy, Utsonomiya, Japan, November 2016 